\title{
Aberrant Maturation of the Uncinate Fasciculus Follows Exposure to Unpredictable Patterns of Maternal Signals
}

\author{
Steven J. Granger ${ }^{1,2}$ Laura M. Glynn, ${ }^{3}$ Curt A. Sandman, ${ }^{4}$ Steven L. Small, ${ }^{5}$ Andre Obenaus, ${ }^{6}$ David B. Keator, \\ Tallie Z. Baram, ${ }^{1,6,7}$ Hal Stern, ${ }^{8}$ Michael A. Yassa, ${ }^{1,2,4}$ and Elysia Poggi Davis ${ }^{4,9}$ \\ ${ }^{1}$ Center for the Neurobiology of Learning and Memory, University of California, Irvine, California 92697, ${ }^{2}$ Department of Neurobiology and \\ Behavior, University of California, Irvine, California 92697, ${ }^{3}$ Department of Psychology, Chapman University, Orange, California 92866, \\ ${ }^{4}$ Department of Psychiatry and Human Behavior, University of California, Irvine, California 92697, ${ }^{5}$ School of Behavioral and Brain Sciences, \\ University of Texas at Dallas, Richardson, Texas 75080, ${ }^{6}$ Department of Pediatrics, University of California, Irvine, California 92697, ${ }^{7}$ Department \\ of Anatomy and Neurobiology, University of California, Irvine, California 92697, ${ }^{8}$ Department of Statistics, University of California, Irvine, \\ California 92697, and ${ }^{9}$ Department of Psychology, University of Denver, Denver, Colorado 80208
}

Across species, unpredictable patterns of maternal behavior are emerging as novel predictors of aberrant cognitive and emotional outcomes later in life. In animal models, exposure to unpredictable patterns of maternal behavior alters brain circuit maturation and cognitive and emotional outcomes. However, whether exposure to such signals in humans alters the development of brain pathways is unknown. In mother-child dyads, we tested the hypothesis that exposure to more unpredictable maternal signals in infancy is associated with aberrant maturation of corticolimbic pathways. We focused on the uncinate fasciculus, the primary fiber bundle connecting the amygdala to the orbitofrontal cortex and a key component of the medial temporal lobe-prefrontal cortex circuit. Infant exposure to unpredictable maternal sensory signals was assessed at 6 and 12 months. Using high angular resolution diffusion imaging, we quantified the integrity of the uncinate fasciculus using generalized fractional anisotropy (GFA). Higher maternal unpredictability during infancy presaged greater uncinate fasciculus GFA in children 9-11 years of age $(n=69,29$ female). In contrast to the uncinate, GFA of a second corticolimbic projection, the hippocampal cingulum, was not associated with maternal unpredictability. Addressing the overall functional significance of the uncinate and cingulum relationships, we found that the resulting imbalance of medial temporal lobe-prefrontal cortex connectivity partially mediated the association between unpredictable maternal sensory signals and impaired episodic memory function. These results suggest that unbalanced maturation of corticolimbic circuits is a mechanism by which early unpredictable sensory signals may impact cognition later in life.

\section{Significance Statement}

Our prior work across species demonstrated that unpredictable patterns of maternal care are associated with compromised memory function. However, the neurobiological mechanisms by which this occurs in humans remain unknown. Here, we identify an association of exposure to unpredictable patterns of maternal sensory signals with the integrity of corticolimbic circuits involved in emotion and cognition using state-of-the-art diffusion imaging techniques and analyses. We find that exposure to early unpredictability is associated with higher integrity of the uncinate fasciculus with no effect on a second corticolimbic pathway, the cingulum. The resulting imbalance of corticolimbic circuit development is a novel mediator of the association between unpredictable patterns of maternal care and poorer episodic memory.

Received Feb. 17, 2020; revised Nov. 25, 2020; accepted Dec. 2, 2020.

Author contributions: S.J.G., L.M.G., C.A.S., S.L.S., T.Z.B., M.A.Y., and E.P.D. designed research; S.J.G., L.M.G., C.A.S., S.L.S., A.O., and E.P.D. performed research; A.O., D.B.K., and H.S. contributed unpublished reagents/analytic tools; S.J.G. analyzed data; S.J.G., M.A.Y., and E.P.D. wrote the paper.

This work was supported by the National Institute of Health (Grant P50-MH-096889 (Principal Investigator: T.Z.B.); Grant R01-MH-102392 (Principal Investigator: M.A.Y.); Grant R03-MH-086062 (Principal Investigator: E.P.D.); Grant NS-41298 (Principal Investigator: C.A.S.); Grant HD-51852 (Principal Investigator: C.A.S.); and Grant HD-28413 (Principal Investigator: C.A.S.).

The authors declare no competing financial interests.

Correspondence should be addressed to Michael A. Yassa at myassa@uci.edu or Elysia Poggi Davis at Elysia.Davis@du.edu.

https://doi.org/10.1523/JNEUROSC1.0374-20.2020

Copyright $\odot 2021$ the authors

\section{Introduction}

Experiences occurring during sensitive periods in early life are powerful factors influencing brain development and cognition (Hasler et al., 2004; Andersen and Teicher, 2008; Short and Baram, 2019). Although it is clear that the quality of maternal care affects the risk for offspring psychological disorders and neurobiological changes later in life (Bowlby, 1950; Lebel et al., 2016; Glynn and Baram, 2019), less is known about how patterns of maternal behavior impact human development (Baram et al., 2012; Davis et al., 2017). Recent work has indicated that 
unpredictable patterns of maternal sensory signals during infancy are a novel contributor to impaired cognitive and emotional functions later in life (Chen and Baram, 2016; Molet et al., 2016b; Walker et al., 2017; Glynn and Baram, 2019; Noraña-Zhou et al., 2020).

We previously reported in both humans and rodents that exposure to greater unpredictable sensory signals during infancy is associated with memory deficits later in life (Ivy et al., 2008, 2010; Molet et al., 2016a; Bath et al., 2017; Davis et al., 2017). In rodents, pup exposure to unpredictable maternal behavior leads to abnormal maturation of brain circuits involved with memory (Brunson et al., 2005; Ivy et al., 2010; Guadagno et al., 2016; Molet et al., 2016a; Walker et al., 2017). Whether the same corticolimbic circuits are impacted by unpredictable maternal sensory signals in humans remains unknown.

Across species, aberrant maturation of amygdala-prefrontal circuits is well recognized as a key outcome of exposure to stress (Burghy et al., 2012; Gee et al., 2013; Chen and Baram, 2016; Tottenham and Galván, 2016; Burgos-Robles et al., 2017). Accelerated maturation of amygdala-prefrontal cortex (PFC) functional connectivity may be an adaptive response to early life adversity, which reprioritizes developmental goals to match the demands of adverse early life environments (Gee et al., 2013). In humans, the predominant anatomic connection between these two regions is the uncinate fasciculus (UF; Ebeling and von Cramon, 1992). The uncinate is thought to be important for episodic memory and has recently been hypothesized to play an important role in adjudicating among competing memory representations during retrieval (Von Der Heide et al., 2013; Alm et al., 2016). Abnormal development of the uncinate could lead to impairments in cognitive and emotional functions (Carballedo et al., 2012; Zhang et al., 2012; Vilgis et al., 2017; Yang et al., 2017; Bhatia et al., 2018). Abnormal development of the uncinate has been associated with early life adversity including a history of institutionalization during childhood and paternal depressive symptoms (Eluvathingal et al., 2006; Govindan et al., 2010; Hanson et al., 2015; Ho et al., 2017; El Marroun et al., 2018).

Here, we evaluated whether exposure to unpredictable maternal signals in infancy is associated with uncinate integrity, measured between the ages of 9 and 11 years. To determine whether the observed effects were specific to the uncinate or are general features of corticolimbic white matter, we conducted similar analyses in the hippocampal cingulum, which shares the frontotemporal connectivity of the uncinate but is spatially distinct (Bubb et al., 2018). As the functional output of brain circuits is a combinatorial sum of the activity of each of their components (Redish and Gordon, 2017), we also conducted analyses using the ratio of uncinate to hippocampal cingulum connectivity as a measure of imbalance across these two pathways.

To determine the behavioral relevance of changes in white matter connectivity associated with unpredictable maternal sensory signals, we focused on episodic memory function, which we assessed by using the mnemonic similarity task (MST), a well validated task (Stark et al., 2019) that is sensitive to the ability of the hippocampus to discriminate among highly similar experiences (i.e., pattern separation), which is thought to be a core facet of episodic memory (Marr, 1971; Yassa and Stark, 2011; Leal and Yassa, 2018; Leutgeb et al., 2007). This task was chosen as we have previously shown across species that greater unpredictability of maternal sensory signals in infancy is associated with differences in memory function (Davis et al., 2017). Further, MST performance is dependent on successful
Table 1. Sample characteristics for mother/infant dyads $(N=73)$

\begin{tabular}{ll}
\hline Characteristics & Values \\
\hline Maternal characteristics & \\
$\quad$ Cohabitation status (\% married or cohabitating) & 84.9 \\
$\quad$ Education, years (SE) & $16(0.4)$ \\
Education (\%) & \\
$\quad$ High school or less & 11.1 \\
Some college, associate degree, or vocational degree & 28.7 \\
4 year college degree & 28.8 \\
Graduate degree & 23.3 \\
Maternal race/ethnicity (\%) & \\
White/European/North African/Middle Eastern & 49.3 \\
Asian & 12.3 \\
Hispanic white & 30.1 \\
Multiethnic/other & 8.2 \\
Maternal depression/anxiety (mean \pm SE) & \\
STAl & $17.2222 \pm 0.641$ \\
EPDS & $4.95 \pm 0.612$ \\
Maternal sensitivity (mean \pm SE) & $9.85 \pm 0.12$ \\
Income to needs ratio (mean \pm SE) & $509.36 \pm 59.5$ \\
Maternal unpredictable sensory signals (mean \pm SE) & $0.81 \pm 0.018$ \\
Age at MRI scan (mean \pm SE) & $9.92 \pm 0.078$ \\
Sex & 43 males, 30 females \\
Days between scan and MST completion & $99 \pm 12.14$ \\
\hline
\end{tabular}

adjudication among competing memory representations during retrieval, which is thought to be a core function of the uncinate fasciculus (Von Der Heide et al., 2013; Alm et al., 2016).

\section{Materials and Methods}

Participants

A sample of 73 mother-offspring dyads ( $n=30$ females) from a prospective, longitudinal, prenatal cohort participated. Initial maternal recruitment criteria included the following: English-speaking, $>18$ years old, nonsmokers, no evidence of drug/alcohol use, and singleton pregnancy. Unpredictable maternal sensory signals were assessed when the infants were 6 and 12 months of age, and the children participated in a single MRI scan between 9 and 11 years of age. Demographic information for the full sample of participants is summarized in Table 1.

\section{Measurement of unpredictability of maternal sensory signals}

Coding maternal sensory signals. Unpredictable maternal sensory signals were coded from a free play dyadic interaction between a mother and her child, as described in the study by Davis et al. (2019). Briefly, mothers were video recorded interacting with their child in a semistructured 10 min play episode at 6 and 12 months of age. Mothers were given a standard set of age-appropriate toys and instructed to interact with their infants naturally as if they were at home. Behaviors from the mother that provided auditory, visual, or tactile sensory signals to the child were coded on a moment-to-moment basis from the video recordings using The Observer XT 11 (Noldus). Auditory signals included all maternal vocalizations, visual signals included maternal manipulation of toy or object while the infant was visually attending, and tactile signals involved all instances of physical contact (holding/touching) initiated by the mother. Coders were blind to all other information on study participants. Interrater reliability was calculated based on $20 \%$ of videos and averaged 89\% (Davis et al., 2017).

Quantifying unpredictability of maternal sensory signals. Unpredictability of maternal sensory signals were quantified by calculating the entropy rate of the sequences of visual, tactile, and auditory maternal signals assessed during the two $10 \mathrm{~min}$ naturalistic observation periods described above. The entropy rate is determined as follows. We first identify all transitions among visual, tactile, and auditory maternal signals, accounting for all possible combinations of the different types of sensory signals. As an example, if a mother was speaking (auditory) to her child and then shows her a toy (visual) while labeling it (auditory), this would be classified as a transition from auditory only to auditory 
and visual. The resulting transitions are then modeled as changes in the state of a discretestate first-order Markov process and the entropy rate of that process calculated as described in the study by Davis et al. (2017). We considered alternative strategies for calculating the entropy rate (e.g., based on other Markov chain models; second order and third order) as well as a nonparametric approach based on theoretical results relating data compression to unpredictability of maternal sensory signals and found that results were highly consistent (Spearman's rank correlations for resulting unpredictability measures ranging from 0.91 to 0.98 ; Vegetabile et al., 2019). The entropy rate measurement can be thought of as capturing how random or uncertain a mother's next behavior would seem to someone making a guess based on previous observed behavior. Entropy rate can range from 0 (transitions are perfectly predictable, e.g., speech is always followed by touch) to a maximum of 2.807 (unpredictable, the next observed behavior among them appears to occur at random). The resulting unpredictability rates were calculated separately for each visit (at 6 and 12 months) and averaged. A comprehensive description of the calculation regarding maternal unpredictability and the entropy rate quantification is described in the study by Davis et al. (2019), and an R package for the calculation of entropy is available at https://contecenter.uci.edu/ shared-resources.

Episodic memory: mnemonic similarity task. The MST was administered on average $99 \pm 12.13 \mathrm{~d}$ from the date of the MRI scan. The MST consists of the following two phases: an incidental encoding phase and a surprise memory test (Fig. 1). During the incidental encoding phase, children are presented with a series of objects appearing on the screen for $2.0 \mathrm{~s}$ (interstimulus interval, $0.5 \mathrm{~s}$ ). At this time, the children were asked to rate the objects as being found "indoor" or "outdoor" via computer keyboard presses. A total of 64 images was presented during the testing phase. During the retrieval (testing) portion, children were presented with 32 target images (identical images that appeared during encoding), 32 foil images (images that did not appear during encoding), and 32 lure images (variants of images presented during encoding). During the test, children were asked to indicate whether the presented object was "old" (they had seen the image before), "similar" (the images were slightly different from images presented during the test), or "new" (images were novel and not seen during the test). Lure items were varied by similarity ratings on a scale from 1 to 5 with 1 being the most similar (bin 1: most difficult to discriminate) and 5 being the least similar (bin 5: easier to discriminate). Lure items were distributed across bin similarity in the following way: bin 1 (6 items), bin 2 (7 items), bin 3 (7 items), bin 4 (6 items), and bin 5 (6 items). As measures of performance, we calculated a lure discrimination index (LDI), which is defined as the probability of indicating similar when given a lure minus the probability of indicating similar given a foil. In this fashion, this measure controls for response bias (indicating similar for every item). The LDI is used as the index of episodic memory. We analyzed the lowsimilarity conditions (average, L4 and L5) as previous research using the MST demonstrated that children this age exhibit chance-level performance on the more difficult high-similarity conditions (Rollins and Cloude, 2018). A significant advantage of the MST task is the concurrent ability to assess traditional recognition memory by evaluating the recognition of target items versus novel foils. We used performance on this recognition condition as a control to determine the specificity of the relationship between hippocampal pattern separation and unpredictable maternal sensory signals.
Retrieval old/new/similar?

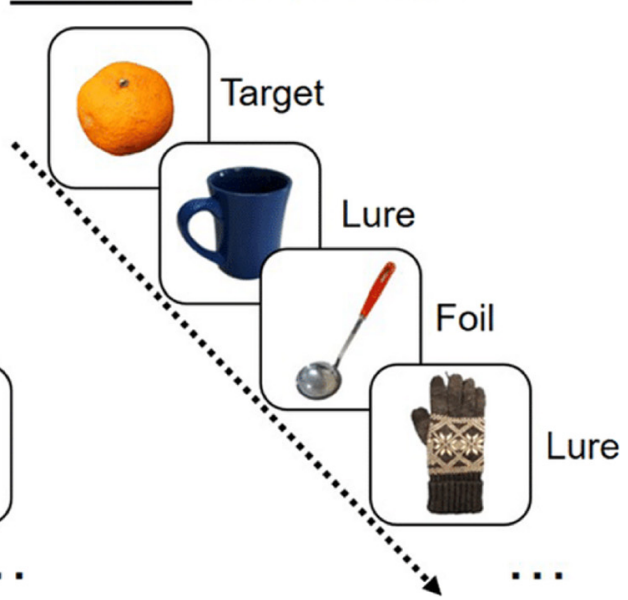

Figure 1. The MST consists of two phases: an incidental encoding phase and a retrieval (testing) phase. The incidental encoding phase consists of a series of 64 images where children are asked to indicate whether the items would appear indoor or outdoor. Upon completion of the incidental encoding phase, children completed the retrieval or testing portion of the MST. During test, children were presented with 32 target images (identical images that appeared during encoding), 32 foil images (images that did not appear during encoding), and 32 lure images (variants of images presented during encoding). Children were asked to indicate whether the presented object was "old" (they had seen the image before), similar (the Lure items were varied by similarity ratings on a scale from 1 to 5 , with 1 being the most similar (bin 1: most difficult to dissimilar (bin 5: easier to discriminate).

Measurement of possible confounding variables.

To assess whether associations with unpredictable maternal sensory signals are influenced by confounding factors, we evaluated maternal mental health, quality of maternal care, and household income. These variables are further described below.

Maternal depression/anxiety. Symptoms of maternal depression and anxiety were assessed at the two postnatal visits (at 6 and 12 months). The Edinburgh Postnatal Depression Scale (EPDS; Cox et al., 1987) and State Anxiety Inventory (STAI; Spielberger et al., 1983) were administered. Scores were standardized and then averaged as an index of maternal mental health (Davis et al., 2019).

Quality of maternal care: maternal sensitivity. To determine whether the unpredictability of maternal signals was associated with brain outcomes beyond more standard measures of quality of maternal care, we evaluated a measure of maternal sensitivity during the same observation period as the quantification of maternal unpredictability. Maternal sensitivity was coded using a protocol developed for the Eunice Kennedy Shriver National Institute of Child Health and Human Development (NICHD) Study of Early Child Care and Youth Development (NICHD Early Child Care Research Network, 1999). Maternal behavior was evaluated for sensitivity to nondistress, intrusiveness (reverse scored), and positive regard ( $1=$ not at all characteristic to $4=$ highly characteristic). A sum of ratings of sensitivity were calculated and used according to the NICHD Early Child Care Research Network (1999).

Income-to-needs ratio. The income-to-needs ratio (INR) was determined by comparing household income to the federal poverty line for a given year and a given household size. Ratios $<1.00$ indicate that household income is below the federal poverty line; in contrast, a ratio of $\geq 1.00$ indicates income above the federal poverty level (Grieger et al., 2009).

\section{Diffusion imaging protocol and processing}

MRI were collected on a 3.0 tesla Philips Achieva scanner at the Neuroscience Imaging Center at the University of California, Irvine, using a 32-channel head coil. The diffusion-weighted imaging scheme consisted of six separate runs of 11 volumes (including one $b=0$ volume) each. The first two runs were collected using a b value of $500 \mathrm{~s} / \mathrm{mm}^{2}$, the third and fourth runs were collected using a b value of $1000 \mathrm{~s} / \mathrm{mm}^{2}$, and the fifth and sixth runs were collected using a b value of $2000 \mathrm{~s} / \mathrm{mm}^{2}$. Thus, the final scan consisted of a multishell 
A

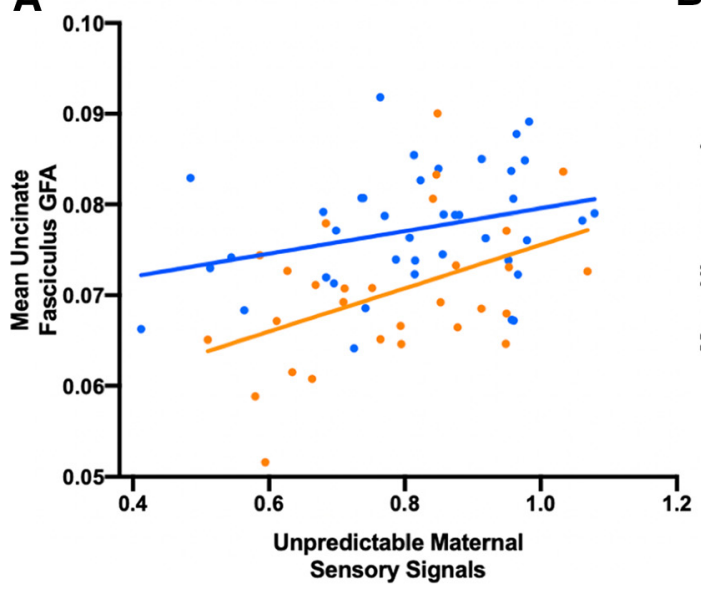

B

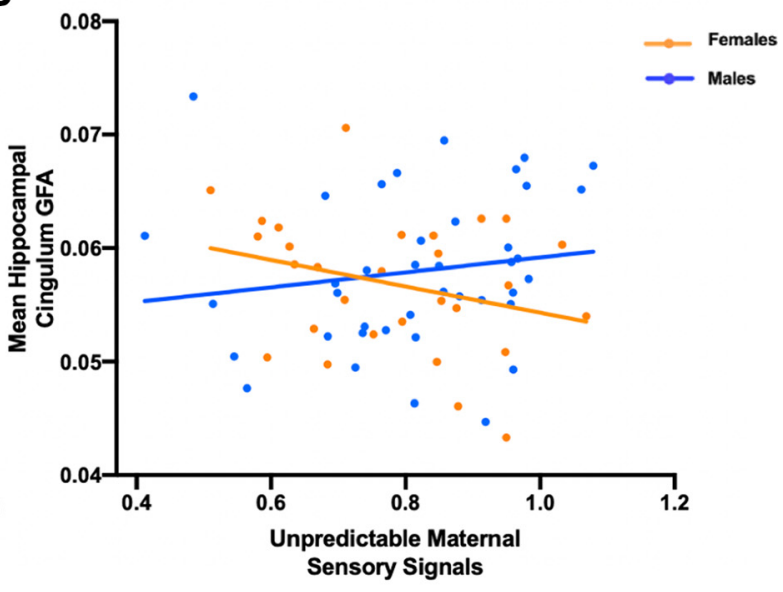

Figure 2. Generalized fractional anisotropy results. $A, A$ significant positive association between exposure to unpredictable maternal sensory signals during infancy and mean uncinate fasciculus GFA primarily in girls $(r=0.44, p=0.016) 9-11$ years of age. This association was not significant in boys $(r=0.30, p=0.057)$. $\boldsymbol{B}$, No association between exposure to unpredictable maternal sensory signals during infancy and mean hippocampal cingulum GFA in girls $(r=-0.29, p=0.13)$ or boys $(r=0.15, p=0.35) 9-11$ years of age.

sequence with 60 noncollinear directions and six B0 volumes. The following scanning parameters were used: direction of acquisition was anterior to posterior, the field of view was 216 (Anterior-Posterior), 216 (Foot-Head), 151.2 (Right-Left); the scan resolution was 108 (10), $127(\mathrm{y})$; number of slices $=84$; voxel size $=1.69 \times 1.68 \times 1.68 \mathrm{~mm}$; $\mathrm{TR}=\sim 11502.76$; $\mathrm{TE}=89$. Raw data were corrected for motion and eddy currents using FSLs eddy program (Andersson and Sotiropoulo, 2016). Data were analyzed using DSI-Studio (http://dsi-studio. labsolver.org; July 26, 2017 build). Corrected data were then reconstructed using the DSI-Studio Q-spin Diffeomorphic Reconstruction (QSDR) function, which uses a powerful diffeomorphic algorithm to warp model-free orientation distribution functions (ODFs) to Montreal Neurologic Institute (MNI) space (Yeh and Tseng, 2011). ODFs were reconstructed with the default diffusion sampling length ratio of 1.25 , which was sufficient to model crossing fibers at the intersection of the corticospinal tract and corpus callosum. This value was additionally validated by varying the diffusion sampling length ratio at intervals of 0.2 and visually examining this intersection using generalized q-sampling imaging. Other reconstruction parameters included the following registration method: norm 7-9-7, eightfold ODF tessellation, number of fibers resolved (5). Output resolution was increased to $1 \mathrm{~mm}$. Quality control for fit to the template space was implemented by setting a criterion on $R^{2}$ at $>0.6$. Four subjects of the total of 73 were eliminated based on these criteria, leaving a final sample size of 69 subjects ( 29 females). Subject head motion was assessed by the eddy_movement_rms file exported from eddy (movement relative to the previous volume) and included as a nuisance regressor. Subject T1-weighted images were used for the quantification of intracranial brain volume. Structural MPRAGE scans were collected at the time of the diffusion scan with the following parameters: $1 \mathrm{~mm}$ isotropic resolution; 208 sagittal slices; field of view $=208 \times$ $256 \times 256 \mathrm{~mm}$; flip angle $=8^{\circ} ; \mathrm{TR}=8 \mathrm{~ms} ; \mathrm{TE}=3.7 \mathrm{~ms}$; matrix size $=$ $256 \times 210 \mathrm{~mm}$. Intracranial volume was calculated using Freesurfer version 6.0 and used to rule out whether differences in GFA were because of intracranial volume.

Region of interest-based approach

Masks of regions of interest (ROIs) were obtained through the Johns Hopkins white matter atlas (in MNI space), which is available within DSI Studio. Regions included limbic white matter regions, namely, the uncus of the uncinate fasciculus and the hippocampal cingulum bilaterally. Averaged generalized fractional anisotropy (GFA) values were extracted for each ROI and averaged across hemispheres. GFA is one of several model-free diffusion measures and is known to correlate with fractional anisotropy of the tensor model. GFA was used to assess the structural integrity of complex tissues in a clinical setting, particularly when there are heterogeneous fiber tissues (Koh et al., 2018; Yamada et al., 2018), and has been used in studies of affective disorders (Chiang et al., 2016; Lo et al., 2017). However, it should be noted that the accuracy of GFA depends on the type of tissue being evaluated as well as the $b$ value at which the raw data are collected (Tuch et al., 2002; Tuch, 2004; Gorczewski et al., 2009; Fritzsche et al., 2010).

\section{Statistical analysis}

Covariates were identified based on theoretical links to either maternal behavior or child outcomes. Covariates included in all analyses were child sex, maternal depressive and anxiety symptoms, maternal sensitivity, and income-to-needs ratio. Correlational analysis was conducted using Prism GraphPad 7 with two-tailed tests of Pearson correlation coefficients. Multiple linear regression models were implemented using RStudio to assess the association between unpredictable maternal sensory signals and the integrity of the white matter tract (uncinate fasciculus and hippocampal cingulum) after the consideration of covariates. Mediation analyses were conducted using the "mediation" package in RStudio. Each mediation model was tested using bias-corrected and accelerated procedures with 10,000 simulations. One case of missing data were imputed for the measurement of maternal depression and anxiety using all possible covariates and uncinate fasciculus GFA (Sinharay et al., 2001). Three cases of missing data were imputed for INR in a similar manner using all possible covariates (including maternal depression/anxiety and uncinate fasciculus GFA).

\section{Results}

Exposure to unpredictable maternal sensory signals during infancy is associated with higher uncinate fasciculus integrity at age 9-11 years

First, we tested the hypothesis that exposure to unpredictable maternal sensory signals in infancy is associated with the integrity of the uncinate fasciculus in 9- to 11-year-old children using GFA as our primary outcome measure. Exposure to unpredictable maternal sensory signals during infancy predicted greater UF GFA ( $r=0.37, p=0.0017$; Fig. $2 a)$. Multiple regressions with covariates (maternal depression/anxiety, maternal sensitivity, income-to-needs ratio, and sex) did not change the association between unpredictable maternal sensory signals and UF GFA ( $\beta=0.26, R^{2}=0.35, p=0.020$; Table 2$)$. Sex was significant as an independent predictor of UF GFA. However, the sex by unpredictability interaction was nonsignificant.

To rule out the possibility that age at scan and in-scanner head motion (derived from $e d d y$ ) were responsible for differences 
Table 2. Unpredictability of maternal sensory signals related to increased uncinate fasciculus GFA accounting for possible covariates

\begin{tabular}{|c|c|c|c|c|c|c|}
\hline Regression model & $R^{2}$ & $F$ & $\beta$ & Standardized $\beta$ & $\mathrm{SE}(\beta)$ & $\overline{p \text { value }}$ \\
\hline Overall model & 0.35 & 6.73 & & & & \\
\hline Unpredictability of maternal sensory signals & & & $1.34 \times 10^{-2}$ & 0.26 & $5.62 \times 10^{-3}$ & 0.020 \\
\hline Maternal depression anxiety & & & $5.054 \times 10^{-4}$ & 0.065 & $7.96 \times 10^{-4}$ & 0.53 \\
\hline Maternal sensitivity & & & $-1.56 \times 10^{-3}$ & -0.21 & $8.041 \times 10^{-4}$ & 0.057 \\
\hline Income-to-needs ratio & & & $-1.01 \times 10^{-6}$ & -0.066 & $1.60 \times 10^{-6}$ & 0.53 \\
\hline Sex & & & $-6.30 \times 10^{-3}$ & -0.39 & $1.67 \times 10^{-3}$ & 0.00036 \\
\hline
\end{tabular}

Table 3. Results of interaction model with unpredictability of hippocampal cingulum GFA predicted by unpredictability of maternal sensory signals, sex, and their interaction

\begin{tabular}{|c|c|c|c|c|c|c|}
\hline Regression model & $R^{2}$ & $F$ & $\beta$ & Standardized $\beta$ & $\operatorname{SE}(\beta)$ & $p$ value \\
\hline Overall model & 0.052 & 1.18 & & & & \\
\hline Unpredictability of maternal sensory signals & & & 0.025 & 0.59 & 0.015 & 0.11 \\
\hline Sex & & & 0.013 & 1.024 & 0.0084 & 0.12 \\
\hline
\end{tabular}

A

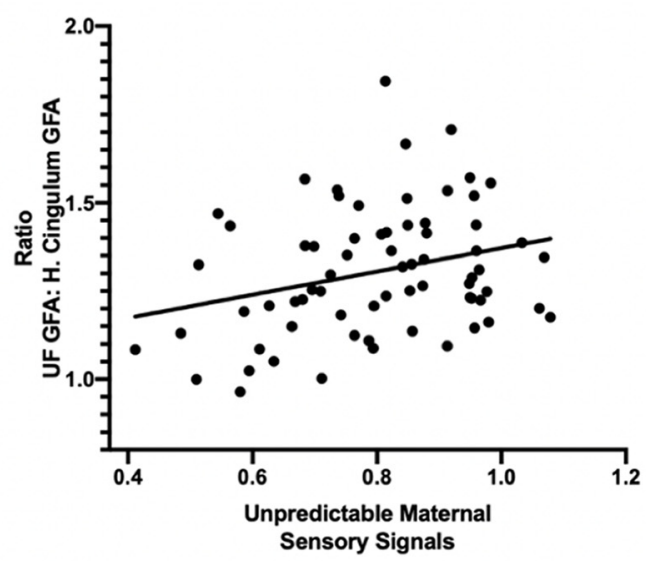

C

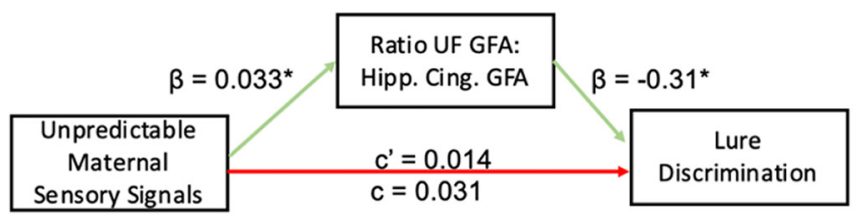

B

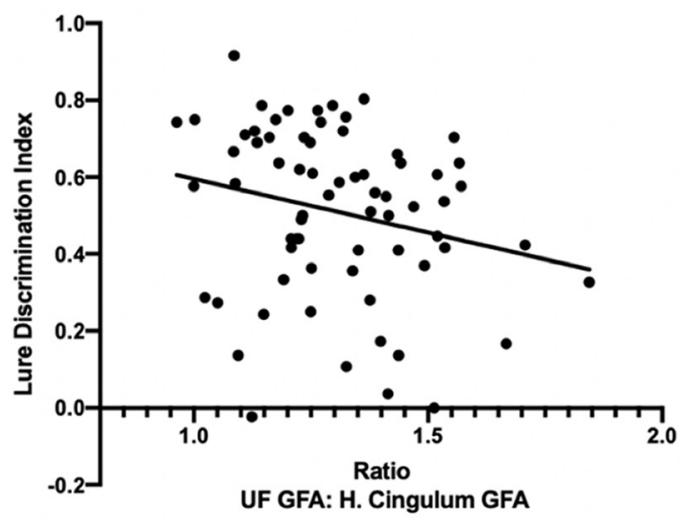

D

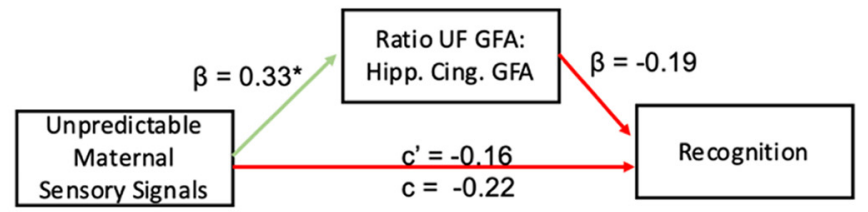

Figure 3. Results of UF/cingulum ratio analyses. $A$, Shows the statistically significant positive association between unpredictable maternal sensory signals and the ratio of anterior MTL-PFC connectivity (uncinate fasciculus GFA) to posterior MTL-PFC connectivity (hippocampal cingulum GFA; $r=0.28, p=0.018$ ). $\boldsymbol{B}$, The marginally significant association between a greater ratio of anterior MTL-PFC connectivity to posterior MTL-PFC connectivity and impaired lure discrimination on low-similarity lure items $(r=-0.23, p=0.058)$. C, $\boldsymbol{D}$, The results of the mediation analyses. $\beta$ values represent the coefficient for the arrows effect. $c^{\prime}$ represents the direct effect, and c represents the total effect of unpredictable maternal sensory signals on low-similarity lure discrimination. C, Shows the significant indirect effect of the ratio of uncinate fasciculus GFA to hippocampal cingulum GFA mediating the association between unpredictable maternal sensory signals and low similarity lure discrimination. $\boldsymbol{D}$, The nonsignificant indirect effect of the ratio of uncinated fasciculus GFA to hippocampal cingulum GFA mediating the association between unpredictable maternal sensory signals and recognition performance is statistically significant at $p<.05$.

in UF GFA, we included them as nuisance regressors in an additional model with all previously mentioned covariates. The association between unpredictable maternal sensory signals and UF GFA ( $\left.\beta=0.26, R^{2}=0.38, p=0.017\right)$ remained significant after accounting for age and head motion. Additionally, neither age, subject head motion, nor their interaction predicted UF GFA. Additionally, intracranial volume did not predict UF GFA $(r=-0.023, p=0.85)$. Finally, including age and intracranial volume (calculated from T1-weighted images) as covariates in our analyses did not alter the statistical significance of the relationships between unpredictable maternal sensory signals and UF GFA.

Because the functional output of brain circuits is a combinatorial sum of the activity of each of their components, we conducted similar analyses in the hippocampal cingulum bundle, a second component of the corticolimbic circuit that connects the connects the medial temporal lobe (MTL) with the PFC via a posterior pathway through the retrosplenial cortex (Bubb et al., 2018). While unpredictable maternal sensory signals did not significantly predict hippocampal cingulum GFA $(r=-0.0026$, $p=0.98$; Fig. $2 b$ ), the sex by unpredictability interaction was marginally significant (Table $3 ; \beta=-1.22, R^{2}=0.052, p=0.086$ ). The directionality of the associations identified in the UF and marginal interaction with sex in the cingulum prompted us to ask whether the two findings are related to an imbalance in the integrity or maturation of these two circuits. We calculated a simple ratio measure of UF/cingulum GFA to capture this imbalance. We found a significant association between the unpredictability of 


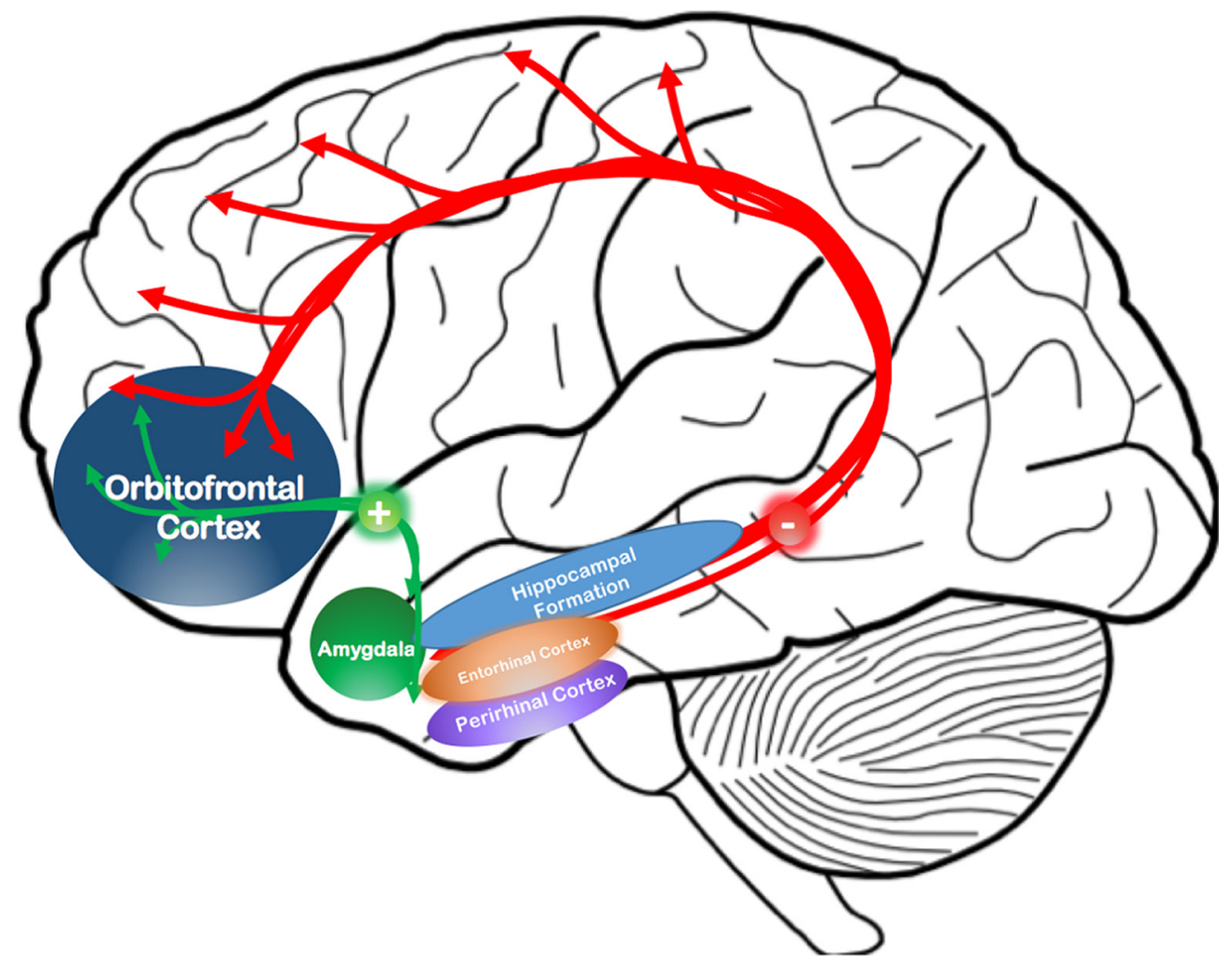

Figure 4. Anterior and posterior MTL-PFC circuits in humans. The circuit schematic summarizes our results demonstrating that (1) the anterior MTL-PFC pathway (i.e., the uncinate fasciculus in green lines), which connects the amygdala and rhinal cortex directly to the orbitofrontal cortex is enhanced in relationship to the increased unpredictability of maternal sensory signals and (2) the posterior MTL-PFC pathway (i.e., the cingulum in red and white), which connects the hippocampus and rhinal cortex with the retrosplenial cortex as well as medial prefrontal and orbitofrontal cortex is not related to the increased unpredictability of maternal sensory signals. These aberrations in circuitry are consistent with a developmental scenario, primarily in female children 9-11 years of age, in which unpredictable maternal sensory signals bias connectivity in favor of the anterior pathway at the expense of the posterior pathway and possibly lead to episodic memory dysfunction.

maternal sensory signals and the ratio of UF GFA to hippocampal cingulum GFA $(r=0.28, p=0.018$; Fig. $3 a)$.

\section{Corticolimbic circuit imbalance is associated with impaired episodic memory performance}

Based on prior work across species (Davis et al., 2017), we hypothesized that the unpredictability of maternal sensory signals would be associated with worse performance on cognitive tasks. In the current study, we used the LDI to assess episodic memory performance. Interestingly, we observed that unpredictable maternal sensory signals did not significantly predict impaired episodic memory performance $(r=0.022, p=0.86)$. We then probed whether the observed circuit changes related to unpredictable maternal sensory signals were associated with episodic memory performance.

UF GFA was not significantly associated with episodic memory performance assessed with the LDI $(r=-0.091, p=0.46)$. However, we found that decreased hippocampal cingulum GFA was marginally associated with lower LDI $(r=0.22, p=0.072)$. A higher UF/cingulum GFA ratio was also marginally associated with lower LDI ( $r=-0.23, p=0.058$; Fig. $3 b)$. The interaction between sex and the UF/cingulum ratio in association with LDI did not reach statistical significance $(\beta=1.28, p=0.13)$.

We observed a significant indirect association through which higher levels of unpredictable maternal sensory signals was associated with a higher UF/cingulum GFA ratio, which, in turn, was associated with worse LDI. In separate mediation models, neither UF GFA (indirect effect $=-0.061, \mathrm{CI}=$ -0.24 to $0.097, p=0.44$ ) nor cingulum GFA (indirect effect $=$
$-0.0008, \mathrm{CI}=-0.088$ to $0.11, p=0.99)$ were significant mediators of the association between unpredictable maternal sensory signals and LDI; however, the UF/cingulum GFA ratio measure was a marginally significant mediator of the association between unpredictable maternal sensory signals and LDI (indirect effect $=-0.10, p=0.049, \mathrm{CI}=-0.28$ to -0.0081 ; Fig. $3 c)$. Critically, this association was specific to lure discrimination and did not generalize to general recognition memory; the indirect association was not statistically significant with the recognition memory outcome (indirect effect $=-0.063$, $p=0.31, \mathrm{CI}=-0.27$ to 0.034 ; Fig. $3 d$ ).

\section{Discussion}

There are three key findings of this study. First, we show that infant exposure to unpredictable patterns of maternal-derived sensory signals is associated with greater integrity of the uncinate fasciculus in late childhood. Second, this finding is specific to the uncinate fasciculus and is not present in the hippocampal cingulum. Last, we find that the ratio of uncinate fasciculus (anterior MTL-PFC connectivity) to hippocampal cingulum GFA (posterior MTL-PFC connectivity; Fig. 4) mediates the association between experiencing greater unpredictability of maternal sensory signals and impaired performance on an episodic memory task. Together, these data suggest that one possible consequence of early life unpredictability is desynchronized maturation of two key corticolimbic pathways resulting in selective impairments in episodic memory tasks requiring nuanced and fine-tuned MTLPFC connectivity. Such findings are consistent with those of 
other studies, suggesting that these connections are vulnerable to early life adversity (Gee et al., 2013; Tottenham and Galván, 2016).

Unpredictable maternal sensory signals have been identified as a novel type of early life adversity, associated with reduced memory and executive functioning (Davis et al., 2017, 2019) an association that has been observed in independent cohorts, such as FinnBrain (Karlsson et al., 2018; Davis et al., 2019). Evidence that patterns of sensory input contribute to the maturation of brain pathways has been previously shown in rodents (Molet et al., 2016a; Bolton et al., 2018) and affects emotional (Walker et al., 2017) as well as cognitive circuits (Chen and Baram, 2016). Here we provide evidence that corticolimbic circuits are associated with infant exposure to unpredictability from the mother in early life and that imbalance among these circuits serves as a possible mechanism explaining differences in cognitive function observed here and in previous studies (Davis et al., 2017, 2019).

Past literature on the association of uncinate fasciculus with various types of early life adversity report mixed results. For example, several studies have found reductions in fractional anisotropy associated with early life maltreatment and deprivation (Eluvathingal et al., 2006; Govindan et al., 2010; Hanson et al., 2015; Ho et al., 2017). In contrast, other studies reported increased diffusion properties of the uncinate fasciculus in premature infants exposed to prenatal maternal stress (Lautarescu et al., 2019) and among adults exposed to childhood trauma (Tatham et al., 2016). Discrepancies may be because of differences in the type of adversity and developmental stage at assessment, and none of these prior studies evaluated unpredictability. We focus on unpredictability as a type of early adversity that has been associated with long-term consequences for development (Glynn et al., 2018, 2019; Howland et al., 2020; Noraña-Zhou et al., 2020). Patterns of sensory signals are known to shape the development of neural circuits involved in sensory systems, and we show that circuits involved in cognitive functions also may be affected. Further, almost all previous studies have quantified the integrity of the uncinate fasciculus using tensor-based measures such as fractional anisotropy, and axial and radial diffusivity. These measures rely on the tensor model, which assumes a single fiber orientation per voxel and does not properly account for fiber crossing, bending, or twisting (Alexander et al., 2001). A strength of our approach of modeling multiple fiber orientations using orientation distribution functions is possibly more likely to approximate microstructural properties of complex neural tissue than the tensor model (Gorczewski et al., 2009; Fritzsche et al., 2010; Yamada et al., 2018).

Anatomically, the uncinate fasciculus is a U-shaped, fanning white matter bundle with complex structure (Ebeling and von Cramon, 1992; Bhatia et al., 2012, 2017, 2018; Riva-Posse et al., 2014; Vergani et al., 2016) and a number of termination points in the medial temporal lobes (Ebeling and von Cramon, 1992; Thiebaut de Schotten et al., 2012; Von Der Heide et al., 2013). It has only recently been visualized using Q-space imaging methods that model the $\mathrm{U}$-shaped uncus and orbitofrontal cortex branching and turning patterns (Leng et al., 2016; Bhatia et al., 2017). Developmental studies have shown that white matter rapidly develops during early infancy, with MTL-PFC pathways like the uncinate (as well as cingulum) continuing to develop until the age of 35 years (Hermoye et al., 2006; Asato et al., 2010; Von Der Heide et al., 2013; Simmonds et al., 2014; Olson et al., 2015). In animal models, a very similar pattern exists as the amygdalaprefrontal connection is relatively slow to develop and continues into adolescence with very rapid growth during the first 10 postnatal days of life followed by synaptic pruning in adolescence (Cunningham et al., 2002; Johnson et al., 2016). Although histologic evidence is needed to truly determine the significance of age-related increased anisotropy across maturation, evidence points to very rapid limbic white matter change in the first 2 years of life, possibly indicating a period of sensitivity in which environmental stimuli could more drastically affect the development of limbic white matter (Yu et al., 2020). In contrast to the association with the uncinate fasciculus, early life exposure to unpredictability was not related to integrity in the cingulum bundle, which is in contrast with previous reports suggesting that this pathway is vulnerable to early life adversity (Choi et al., 2009; Huang et al., 2012; El Marroun et al., 2018).

The function of the uncinate fasciculus is still not well understood, although recent studies have suggested that it plays a role in episodic memory, and in particular the adjudication among similar mnemonic representations during retrieval (Von Der Heide et al., 2013; Alm et al., 2016). It is well established that episodic memory involves the MTL, the PFC, as well as their interactions (Jones and Wilson, 2005; Van Kesteren et al., 2010; Preston and Eichenbaum, 2013; Brincat and Miller, 2015; Eichenbaum, 2017). Further, the ability to adjudicate among competing memory representations is thought to rely on hippocampal pattern separation. With this in mind, our primary behavioral outcome measure (LDI) was based on the mnemonic similarity task, which is designed to assess hippocampal pattern separation. Our results provide evidence that an imbalanced ratio of uncinate connectivity to cingulum connectivity is related to impaired performance on the mnemonic similarity task. Additionally, our mediation analyses suggest that this imbalanced connectivity, a possible indicator of imbalanced maturation, is a putative mechanism that links the experience of unpredictable maternal sensory signals early in infancy to impaired memory function in childhood.

The ratio of integrity parameters of the uncinate fasciculus to cingulum was a mediator in the association between unpredictable maternal sensory signals and performance in our task. This should not be surprising, as both of these projections comprise components of the corticolimbic brain circuitry, which is critical for executing complex behaviors such as memory. Notably, the overall function of the circuit can be thought of as the combinatorial sum of the function and connectivity of its distinct components (Redish and Gordon, 2017). Thus, whereas augmented maturation of a single component or a deficit in another in and by itself may not suffice to distort circuit function, their combined existence may be synergistic, leading to unbalanced functional output.

In conclusion, our findings demonstrate that infant exposure to unpredictable patterns of maternal care is associated with imbalanced maturation of the uncinate fasciculus and the cingulum detected during childhood, and that this imbalance may be associated with deficits in memory function. This is consistent with our prior work in rodents showing that early life unpredictability leads to increased structural connectivity of the amygdala to prefrontal cortex pathway in rats (Bolton et al., 2018) and to deficits in hippocampal memory (Molet et al., 2016b). This study, along with future investigations, will yield novel insight into neurobiological mechanisms of vulnerability to cognitive deficits as a result of unpredictable early environments.

\section{References}

Alexander AL, Hasan KM, Lazar M, Tsuruda JS, Parker DL (2001) Analysis of partial volume effects in diffusion-tensor MRI. Magn Reson Med 45:770-780 
Alm KH, Rolheiser T, Olson IR (2016) Inter-individual variation in frontotemporal connectivity predicts the ability to learn different types of associations. Neuroimage 132:213-224.

Andersen SL, Teicher MH (2008) Stress, sensitive periods and maturational events in adolescent depression. Trends Neurosci 31:183-191.

Andersson JLR, Sotiropoulo SN (2016) An integrated approach to correction for off-resonance effects and subject movement in diffusion MR imaging. Neuroimage 125:1063-1078.

Asato MR, Terwilliger R, Woo J, Luna B (2010) White matter development in adolescence: a DTI study. Cereb Cortex 20:2122-2131.

Baram TZ, Davis EP, Obenaus A, Sandman CA, Small SL, Solodkin A, Stern $H$ (2012) Fragmentation and unpredictability of early-life experience in mental disorders. Am J Psychiatry 169:907-915.

Bath KG, Nitenson AS, Lichtman E, Lopez C, Chen W, Gallo M, Goodwill H, Manzano-Nieves G (2017) Early life stress leads to developmental and sex selective effects on performance in a novel object placement task. Neurobiol Stress 7:57-67.

Bhatia K, Henderson L, Yim M, Hsu E, Dhaliwal R (2017) Diffusion tensor imaging investigation of uncinate fasciculus anatomy in healthy controls: description of a subgenual stem. Neuropsychobiology 75:132-140.

Bhatia KD, Henderson L, Ramsey-Stewart G, May J (2012) Diffusion tensor imaging to aid subgenual cingulum target selection for deep brain stimulation in depression. Stereotact Funct Neurosurg 90:225-232.

Bhatia KD, Henderson LA, Hsu E, Yim M (2018) Reduced integrity of the uncinate fasciculus and cingulum in depression: a stem-by-stem analysis. J Affect Disord 235:220-228.

Bolton JL, Molet J, Regev L, Chen Y, Rismanchi N, Haddad E, Yang DZ, Obenaus A, Baram TZ (2018) Anhedonia following early-life adversity involves aberrant interaction of reward and anxiety circuits and is reversed by partial silencing of amygdala corticotropin-releasing hormone gene. Biol Psychiatry 83:137-147.

Bowlby J (1950) Research into the origins of delinquent behaviour. Br Med J $1: 570-573$.

Brincat SL, Miller EK (2015) Frequency-specific hippocampal-prefrontal interactions during associative learning. Nat Neurosci 18:576-581.

Brunson KL, Kramár E, Lin B, Chen Y, Colgin LL, Yanagihara TK, Lynch G, Baram TZ (2005) Mechanisms of late-onset cognitive decline after earlylife stress. J Neurosci 25:9328-9338.

Bubb EJ, Metzler-baddeley C, Aggleton JP (2018) The cingulum bundle: anatomy, function, and dysfunction. Neurosci Biobehav Rev 92:104-127.

Burghy CA, Stodola DE, Ruttle PL, Molloy EK, Armstrong JM, Oler JA, Fox ME, Hayes AS, Kalin NH, Essex MJ, Davidson RJ, Birn RM (2012) Developmental pathways to amygdala- prefrontal function and internalizing symptoms in adolescence. Nat Neurosci 15:1736-1741.

Burgos-Robles A, Kimchi EY, Izadmehr EM, Porzenheim MJ, Ramos-Guasp WA, Nieh EH, Felix- Ortiz AC, Namburi P, Leppla CA, Presbrey KN, Anandalingam KK, Pagan-Rivera PA, Anahtar M, Beyeler A, Tye KM (2017) Amygdala inputs to prefrontal cortex guide behavior amid conflicting cues of reward and punishment. Nat Neurosci 20:824-835.

Carballedo A, Amico F, Ugwu I, Fagan AJ, Fahey C, Morris D, Meaney JF, Leemans A, Frodl T (2012) Reduced fractional anisotropy in the uncinate fasciculus in patients with major depression carrying the met-allele of the Val66Met brain-derived neurotrophic factor genotype. Am J Med Genet B Neuropsychiatr Genet 159B:537-548.

Chen Y, Baram TZ (2016) Toward understanding how early-life stress reprograms cognitive and emotional brain networks. Neuropsychopharmacology 41:197-206.

Chiang HL, Chen YJ, Shang CY, Tseng WYI, Gau SSF (2016) Different neural substrates for executive functions in youths with ADHD: a diffusion spectrum imaging tractography study. Psychol Med 46:1225-1238.

Choi J, Jeong B, Rohan ML, Polcari AM, Teicher MH (2009) Preliminary evidence for white matter tract abnormalities in young adults exposed to parental verbal abuse. Biol Psychiatry 65:227-234

Cox JL, Holden JM, Sagovsky R (1987) Detection of postnatal depression: development of the 10-item Edinburgh Postnatal Depression Scale. Br J Psychiatry 150:782-786.

Cunningham MG, Bhattacharyya S, Benes FM (2002) Amygdalo-cortical sprouting continues into early adulthood: implications for the development of normal and abnormal function during adolescence. J Comp Neurol 453:116-130.

Davis EP, Stout SA, Molet J, Vegetabile B, Glynn LM, Sandman CA, Hein K, Stern H, Baram TZ (2017) Exposure to unpredictable maternal sensory signals influences cognitive development across species. Proc Natl Acad Sci U S A 114:10390-10395.

Davis EP, Korja R, Karlsson L, Glynn LM, Sandman CA, Vegetabile B, Kataja E, Nolvi S, Sinervä E, Pelto J, Karlsson H, Stern HS, Baram TZ (2019) Across continents and demographics, unpredictable maternal signals are associated with children's cognitive function. EBioMedicine 46:256-263.

Ebeling U, von Cramon D (1992) Topography of the uncinate fascicle and adjacent temporal fiber tracts. Acta Neurochir (Wien) 115:143-148.

Eichenbaum H (2017) Prefrontal-hippocampal interactions in episodic memory. Nat Rev Neurosci 18:547-558.

El Marroun H, Jaddoe VW, White T, Zou R, Muetzel RL, Verhulst FC, Tiemeier H (2018) Prenatal exposure to maternal and paternal depressive symptoms and white matter microstructure in children. Depress Anxiety 35:321-329.

Eluvathingal TJ, Chugani HT, Behen ME, Juhász C, Muzik O, Maqbool M, Chugani DC, Makki M (2006) Abnormal brain connectivity in children after early severe socioemotional deprivation: a diffusion tensor imaging study. Pediatrics 117:2093-2100.

Fritzsche KH, Laun FB, Meinzer HP, Stieltjes B (2010) Opportunities and pitfalls in the quantification of fiber integrity: what can we gain from Q-ball imaging? Neuroimage 51:242-251.

Gee DG, Gabard-durnam LJ, Flannery J, Goff B, Humphreys KL, Telzer EH, Hare TA, Bookheimer SY, Tottenham N (2013) Early developmental emergence of human amygdala-prefrontal connectivity after maternal deprivation. Proc Natl Acad Sci U S A 110:15638-15643.

Glynn LM, Baram TZ (2019) The influence of unpredictable, fragmented parental signals on the developing brain. Front Neuroendocrinol 53: 100736.

Glynn LM, Howland MA, Sandman CA, Davis EP, Phelan M, Baram TZ, Stern HS (2018) Prenatal maternal mood patterns predict child temperament and adolescent mental health. J Affect Disord 228:83-90.

Glynn LM, Stern HS, Howland MA, Risbrough VB, Baker DG, Nievergelt CM, Baram TZ, Davis EP (2019) Measuring novel antecedents of mental illness: the Questionnaire of Unpredictability in Childhood. Neuropsychopharmacology 44:876-882.

Gorczewski K, Mang S, Klose U (2009) Reproducibility and consistency of evaluation techniques for HARDI data. MAGMA 22:63-70.

Govindan RM, Behen ME, Helder E, Makki MI, Chugani HT (2010) Altered water diffusivity in cortical association tracts in children with early deprivation identified with tract-based spatial statistics (TBSS). Cereb Cortex 20:561-569.

Grieger LD, Schoeni RF, Danziger S (2009) Accurately measuring the trend in poverty in the United States using the panel study of income dynamics. J Econ Soc Meas 34:105-117.

Guadagno A, Verlezza S, Long H, Wong TP, Walker CD (2016) The effects of chronic early-life stress on amygdala morphology and function in neonatal rats. Paper presented at Neurobiology of Stress Workshop, Irvine, CA, April.

Hanson JL, Knodt AR, Brigidi BD, Hariri AR (2015) Lower structural integrity of the uncinate fasciculus is associated with a history of child maltreatment and future psychological vulnerability to stress. Dev Psychopathol 27:1611-1619.

Hasler G, Drevets WC, Manji HK, Charney DS (2004) Discovering endophenotypes for major depression. Neuropsychopharmacology 29:1765-1781.

Hermoye L, Saint-Martin C, Cosnard G, Lee S-K, Kim J, Nassogne M-C, Menten R, Clapuyt P, Donohue PK, Hua K, Wakana S, Jiang H, van Zijl PCM, Mori S (2006) Pediatric diffusion tensor imaging: normal database and observation of the white matter maturation in early childhood. Neuroimage 29:493-504.

Ho TC, King LS, Leong JK, Colich NL, Humphreys KL, Ordaz SJ, Gotlib IH (2017) Effects of sensitivity to life stress on uncinate fasciculus segments in early adolescence. Soc Cogn Affect Neurosci 12:1460-1469.

Howland MA, Sandman CA, Davis EP, Stern H, Phelan M, Baram TZ, Glynn LM (2020) Prenatal maternal mood entropy is associated with child neurodevelopment. Emotion. Advance online publication. Retrieved December 17, 2020. doi: 10.1037/emo0000726.

Huang H, Gundapuneedi T, Rao U (2012) White matter disruptions in adolescents exposed to childhood maltreatment and vulnerability to psychopathology. Neuropsychopharmacology 37:2693-2701.

Ivy AS, Brunson KL, Sandman C, Baram TZ (2008) Dysfunctional nurturing behavior in rat dams with limited access to nesting material: a clinically relevant model for early-life stress. Neuroscience 154:1132-1142. 
Ivy AS, Rex CS, Chen Y, Dubé C, Maras PM, Grigoriadis DE, Gall CM, Lynch G, Baram TZ (2010) Hippocampal dysfunction and cognitive impairments provoked by chronic early-life stress involve excessive activation of CRH receptors. J Neurosci 30:13005-13015.

Johnson CM, Loucks A, Peckler H, Thomas A, Janak P, Wilbrecht L (2016) Long-range orbitofrontal and amygdala axons show divergent patterns of maturation in the frontal cortex across adolescence. Dev Cogn Neurosci 18:113-120.

Jones MW, Wilson MA (2005) Theta rhythms coordinate hippocampal-prefrontal interactions in a spatial memory task. PLoS Biol 3:e402.

Karlsson L, Tolvanen M, Scheinin NM, Uusitupa HM, Korja R, Ekholm E, Tuulari J, Pajulo M, Huotilainen M, Paunio T, Karlsson H (2018) Cohort profile: the FinnBrain Birth Cohort Study (FinnBrain). Int J Epidemiol 47:15-16.

Koh C, Tang P, Chen H, Hsu Y, Hsieh C, Tseng WI (2018) Impaired callosal motor fiber integrity and upper extremity motor impairment are associated with stroke lesion location. Neurorehabil Neural Repair 32:602-612.

Lautarescu A, Pecheva D, Nosarti C, Nihouarn J, Zhang H, Victor S, Craig M, Edwards D, Counsell SJ (2019) Maternal prenatal stress is associated with altered uncinate fasciculus microstructure in premature neonates. Biol Psychiatry 87:559-569.

Lebel C, Walton M, Letourneau N, Giesbrecht GF, Kaplan BJ, Dewey D (2016) Prepartum and postpartum maternal depressive symptoms are related to children's brain structure in preschool. Biol Psychiatry 80:859868.

Leng B, Han S, Bao Y, Zhang H, Wang Y, Wu Y, Wang Y (2016) The uncinate fasciculus as observed using diffusion spectrum imaging in the human brain. Neuroradiology 58:595-606.

Leal SL, Yassa MA (2018) Integrating new findings and examining clinical applications of pattern separation. Nat Neurosci 21:163-173.

Leutgeb JK, Leutgeb S, Moser M-B, Moser EI (2007) Pattern separation in the dentate gyrus and CA3 of the hippocampus. Science 315:961-966.

Lo YC, Chen YJ, Hsu YC, Tseng WYI, Gau SSF (2017) Reduced tract integrity of the model for social communication is a neural substrate of social communication deficits in autism spectrum disorder. J Child Psychol Psychiatr 58:576-585.

Marr D (1971) Simple memory: a theory for archicortex. Philos Trans R Soc Lond B Biol Sci 262:23-81.

Molet J, Heins K, Zhuo X, Mei YT, Regev L, Baram TZ, Stern H (2016a) Fragmentation and high entropy of neonatal experience predict adolescent emotional outcome. Transl Psychiatry 6:e702.

Molet J, Maras PM, Kinney-Lang E, Harris NG, Rashid F, Ivy AS, Solodkin A, Obenaus A, Baram TZ (2016b) MRI uncovers disrupted hippocampal microstructure that underlies memory impairments after early-life adversity. Hippocampus 26:1618-1632.

NICHD Early Child Care Research Network (1999) Chronicity of maternal depressive symptoms, maternal sensitivity, and child functioning at 36 months. Dev Psychol 35:1297-1310.

Noraña-Zhou AN, Morgan A, Glynn LM, Sandman CA, Baram TZ, Stern HS, Davis EP (2020) Unpredictable maternal behavior is associated with a blunted infant cortisol response. Dev Psychobiol 62:882-888.

Olson IR, Von Der Heide RJ, Alm KH, Vyas G (2015) Development of the uncinate fasciculus: implications for theory and developmental disorders. Dev Cogn Neurosci 14:50-61.

Preston AR, Eichenbaum H (2013) Interplay of hippocampus and prefrontal cortex in memory. Curr Biol 23:R764-R773.

Redish DA, Gordon JA (2017) Computational psychiatry: a new perspective on mental illness. Cambridge, MA: MIT.

Riva-Posse P, Choi KS, Holtzheimer PE, McIntyre CC, Gross RE, Chaturvedi A, Crowell AL, Garlow SJ, Rajendra JK, Mayberg HS (2014) Defining critical white matter pathways mediating successful subcallosal cingulate deep brain stimulation for treatment-resistant depression. Biol Psychiatry 76:963-969.

Rollins L, Cloude EB (2018) Development of mnemonic discrimination during childhood. Learn Mem 25:294-297.

Short AK, Baram TZ (2019) Early-life adversity and neurological disease: age-old questions and novel answers. Nat Rev Neurol 15:657-669.
Simmonds DJ, Hallquist MN, Asato M, Luna B (2014) Developmental stages and sex differences of white matter and behavioral development through adolescence: a longitudinal diffusion tensor imaging (DTI) study. NeuroImage 92:356-368.

Sinharay S, Stern HS, Russell D (2001) The use of multiple imputation for the analysis of missing data. Psychol Methods 6:317-329.

Spielberger CD, Gorsuch RL, Lushene R, Vagg PR, Jacobs GA (1983) Manual for the State-Trait Anxiety Inventory. Palo Alto, CA: Consulting Psychologists.

Stark SM, Kirwan CB, Stark C (2019) Mnemonic similarity task: A tool for assessing hippocampal integrity. Trends in cognitive sciences 23:938951.

Tatham EL, Ramasubbu R, Gaxiola-Valdez I, Cortese F, Clark D, Goodyear B, Foster J, Hall GB (2016) White matter integrity in major depressive disorder: implications of childhood trauma, 5-HTTLPR and BDNF polymorphisms. Psychiatry Res Neuroimaging 253:15-25.

Thiebaut de Schotten M, Dell'Acqua F, Valabregue R, Catani M (2012) Monkey to human comparative anatomy of the frontal lobe association tracts. Cortex 48:82-96.

Tottenham N, Galván A (2016) Stress and the adolescent brain Amygdalaprefrontal cortex circuitry and ventral striatum as developmental targets. Neurosci Biobehav Rev 70:217-227.

Tuch DS (2004) Q-ball imaging. Magn Reson Med 52:1358-1372.

Tuch DS, Reese TG, Wiegell MR, Makris N, Belliveau JW, Wedeen VJ (2002) High angular resolution diffusion imaging reveals intravoxel white matter fiber heterogeneity. Magn Reson Med 48:577-582.

Van Kesteren MT, Fernández G, Norris DG, Hermans EJ (2010) Persistent schema-dependent hippocampal-neocortical connectivity during memory encoding and postencoding rest in humans. Proc Natl Acad Sci U S A $107: 7550-7555$.

Vegetabile BG, Stout-Oswald SA, Davis EP, Baram TZ, Stern HS (2019) Estimating the entropy rate of finite Markov chains with application to behavior studies. J Educ Behav Stat 44:282-308.

Vergani F, Martino J, Morris C, Attems J, Ashkan K, Dell'Acqua F (2016) Anatomic connections of the subgenual cingulate region. Neurosurgery 79:465-472.

Vilgis V, Vance A, Cunnington R, Silk TJ (2017) White matter microstructure in boys with persistent depressive disorder. J Affect Disord 221:11-16.

Von Der Heide RJ, Skipper LM, Klobusicky E, Olson IR (2013) Dissecting the uncinate fasciculus: disorders, controversies and a hypothesis. Brain 136:1692-1707.

Walker CD, Bath KG, Joels M, Korosi A, Larauche M, Lucassen PJ, Morris MJ, Raineki C, Roth TL, Sullivan RM, Taché Y, Baram TZ (2017) Chronic early life stress induced by limited bedding and nesting (LBN) material in rodents: critical considerations of methodology, outcomes and translational potential. Stress 20:421-448.

Yamada N, Ueda R, Kakuda W, Momosaki R, Kondo T, Hada T, Sasaki N, Hara T, Senoo A, Abo M (2018) Diffusion tensor imaging evaluation of neural network development in patients undergoing therapeutic repetitive transcranial magnetic stimulation following stroke. Neural Plast 2018:3901016.

Yang X-H, Wang Y, Wang D-F, Tian K, Cheung EFC, Xie G-R, Chan RCK (2017) White matter microstructural abnormalities and their association with anticipatory anhedonia in depression. Psychiatry Res Neuroimaging 264:29-34.

Yassa MA, Stark CEL (2011) Pattern separation in the hippocampus. Trends Neurosci 34:515-525.

Yeh FC, Tseng WYI (2011) NTU-90: a high angular resolution brain atlas constructed by q-space diffeomorphic reconstruction. Neuroimage 58: 91-99.

Yu Q, Peng Y, Kang H, Peng Q, Ouyang M, Slinger M, Hu D, Shou H, Fang F, Huang H (2020) Differential white matter maturation from birth to 8 years of age. Cereb Cortex 30:2674-2690.

Zhang A, Leow A, Ajilore O, Lamar M, Yang S, Joseph J, Medina J, Zhan L, Kumar A (2012) Quantitative tract-specific measures of uncinate and cingulum in major depression using diffusion tensor imaging. Neuropsychopharmacology 37:959-967. 